Cita: Baptista, C.; Corte-Real, N.; Regueiras, L.; Seo, G.; Hemphill, M.; Pereira, A.; Dias, C.;

Martinek, T.; Fonseca, A. (2020). Teaching personal and social responsibility after school: A systematic review. Cuadernos de Psicología del Deporte, 20(2), 1-25

\title{
Teaching personal and social responsibility after-school: A systematic review
}

\section{Enseñando responsabilidad personal y social en un contexto extracurricular: Una revisión sistemática}

\section{O Ensino da responsabilidade pessoal e social no contexto extracurricular: Uma revisão sistemática}

\author{
Baptista, C.;1 Corte-Real, N.;1 Regueiras, L.;2 Seo, G.;3 Hemphill, M.;3 Pereira, A.;4 Dias, C.;1 Martinek, \\ T.;3 Fonseca, A.1 \\ ${ }_{1}$ CIFI2D (Centre of Investigation, Formation, Innovation and Intervention in Sport), Faculty of Sport, \\ University of Porto, Portugal;
}

2 Nun'Alvres Institute, Santo Tirso, Portugal;

${ }_{3}$ University of North Carolina of Greensboro, United States of America;

${ }_{4}$ Faculty of Sport Sciences and Physical Education of Coimbra, Portugal.

\begin{abstract}
Background: Teaching Personal and Social Responsibility (TPSR) has been studied and implemented through physical activity in different backgrounds for over three decades. However, there is no systematized review in the literature concerning the after-school context.

Aim: Conducting a systematic review of literature on after-school interventions based on the TPSR model.

Methods: This study was driven by the following research questions: Which were the conclusions regarding the implementation of TPSR in after-school settings? Which research methodologies have been used to assess TPSR in after-school time settings? Which results related to TPSR were reported in after-school time settings? Cochrane protocol guidelines were followed. Papers were selected by two independent researchers, with Cohen's Kappa value of $81 \%$.

Results and discussion: Twenty-seven papers were selected, thirteen of which were reported with high scores. Most interventions were conducted in the USA on community-based after-school programs, lasting more than nine sessions, and led by school staff who prepared physical activities for youngsters from disadvantaged communities. Most of the reported studies resorted to qualitative methodologies. Some gaps were detected, such as lack of systematization of methods, lack of validity and reliability. Personal and social benefits were found. Other results were grouped into leadership, staff-youngsters relationship, values, transference, and impact on staff lives.

We recommend future studies in the after-school context extend to extend to other countries, with more detailed descriptions of the specific used methods.

Keywords: Teaching personal and social responsibility, physical activity, TPSR Alliance, after-school.
\end{abstract}

\section{RESUMEN}




\section{Baptista, Corte-Real, Regueiras, Seo, Hemphill, Pereira, Dias, Martinek, Fonseca}

Contexto: Hace más de tres décadas, el Teaching Personal and Social Responsability (TPSR) ha sido estudiado e implementado, a través de la actividad física, en distintos contextos. No obstante, no ha ninguna revisión sistematizada en literatura en un contexto extracurricular.

Objetivo: Llevar a una revisión sistemática de la literatura sobre intervenciones extracurriculares basadas en el modelo TPSR.

Métodos: Las preguntas de búsqueda que llevaron a este estudio fueron: ¿Cuáles las conclusiones relacionadas a la implementación del TPSR en contexto extracurricular? ¿Qué metodologías de investigación han sido utilizadas para examinar el TPSR en contexto extracurricular? ¿Qué resultados relacionados con el TPSR fueron presentados en contexto extracurricular? Han sido seguidas las orientaciones del protocolo de Cochrane. La selección de los artículos ha sido hecha por dos investigadores independientes, con valor de Kappa de Cohen de $81 \%$.

Resultados y discusión: Han sido seleccionados veintisiete artículos, trece de los cuales han sido relatados con elevada puntuación. La mayoría de las intervenciones han sido hechas en los EEUU, en programas extracurriculares para la comunidad, con una duración superior a nueve sesiones, encabezadas por staff de la escuela prepararon actividades físicas para jóvenes de comunidades desfavorecidas. Los estudios relatados apelaron, en su mayoría, a metodologías cualitativas. Algunos errores han sido detectados como la falta de sistematización de los métodos, la falta de validez y fiabilidad. Han sido encontrados beneficios personales y sociales. Otros resultados han sido agrupados en liderazgo, relaciones entre staff y jóvenes, valores, transferencia e impacto en la vida de las personas. Se recomienda que estudios futuros en el contexto extracurricular se extendan a otros países, con descripciones más detalladas de los métodos utilizados y exactos.

Palabras clave: Modelo de responsabilidad, actividad física, TPSR Alliance, extracurricular.

\section{RESUMO}

Contexto: Há mais de três décadas, o Teaching Personal and Social Responsibility (TPSR) tem sido estudado e implementado, através da atividade física, em diferentes contextos. No entanto, não há nenhuma revisão sistematizada na literatura em contexto extracurricular.

Propósito: Conduzir uma revisão sistemática da literatura sobre intervenções extracurriculares baseadas no modelo TPSR.

Métodos: As perguntas de pesquisa que conduziram este estudo foram: quais as conclusões relacionadas à implementação do TPSR em contextos extracurriculares? Que metodologias de investigação têm sido utilizadas para examinar o TPSR em contextos extracurriculares? Que resultados relacionados com o TPSR foram reportados em contextos extracurriculares? Foram seguidas as orientações do protocolo de Cochrane. A seleção dos artigos foi feita por dois pesquisadores independentes, com valor de Kappa de Cohen de $81 \%$.

Resultados e discussão: Foram selecionados vinte e sete artigos, treze dos quais foram relatados com elevada pontuação. A maioria das intervenções foi realizada nos EUA, em programas extracurriculares para a comunidade, com uma duração superior a nove sessões, lideradas por staff da escola que preparou atividades físicas para jovens de comunidades desfavorecidas. Os estudos relatados recorreram, na sua maioria, a metodologias qualitativas. Algumas lacunas foram detetadas como a falta de sistematização dos métodos, a falta de validade e confiabilidade. Foram encontrados benefícios pessoais e sociais. Outros resultados foram agrupados em liderança, relacionamento entre staff e jovens, valores, transferência e impacto na vida pessoal.

Recomenda-se que estudos futuros no contexto extracurricular se estendam para outros países, com descrições mais detalhadas dos métodos usados e precisos.

Palavras chave: Modelo de responsabilidade, atividade física, TPSR Alliance, extracurricular 


\section{Personal and social responsibility model systematic review}

\section{INTRODUCTION}

The Teaching Personal and Social Responsibility (TPSR) model was created by Hellison (1985), based on the assumption that responsibility behaviors can be taught within the contexts of physical activity and can help youngsters adapting to transitions into adulthood.

Hellison (1995, 2003, 2011) proposed a pedagogical program based on the two following assumptions: the first assumption postulates that the instruction of life skills and values is a part of physical activity. The second assumption states that values learned in the classroom should be transferred to after-school settings.

Education values aim youngsters in sports to acquire life-appropriate behaviors, knowledges and attitudes, such as respect, tolerance and fair-play (Díaz, Manzano, Martín, Catalán \& Palacios, 2018).

To achieve these values, youngsters should outperform progressively based on five levels of responsibility (Hellison, 2011): (1) respect for the rights and feelings of others (e.g., asking a student to referee a game during a session); (2) participation and effort (e.g., set achievable goals, as asking students to do five more push-ups than in the previous class, during a session); (3) self-direction (e.g., working by stations, where the instructor dedicates more time in one of the stations and the participants work independently on the other ones, during a session); (4) leadership and helping others (e.g., during a session in heterogeneous teams the ball has to touch every participant before scoring a goal) (5) transference outside the gym (e.g., asking a participant during a session to give an example of respect for others at home or in sports activities with the participants to the community). TPSR-based lessons consist of a typical format, described in detail in Hellison (2011, p. 27): a) relational time: a brief time in which the instructor interacts with participants and mentions something special to them; b) awareness talk: a more formal moment in which the instructor has a brief conversation about the responsibility levels that will be developed in the classroom and sets concrete goals; c) physical activity plan: it occupies most of the time, and all tasks have connected levels of responsibility; d) group meeting: a few minutes before finishing the session, students can express their views on the session activities and how they can be improved; e) self-reflection time: before finishing the session, students can assess how was your performance on personal and social responsibility.

The TPSR model has had a positive impact on the individual behaviors, perceptions and attitudes of participants (Hellison \& Martinek, 2006; Prieto, Delgado, Caro \& Preciado, 2015). This model has been developed in different areas, such as Physical Education (PE), community programs, summer camps, and after-school sports programs (Lee \& Choi, 2015; Walsh, Veri \& Willard, 2015; Wright, Jacobs, Ressler, \& Jung, 2016), as well as in Professional Development for Teachers (Camerino, ValeroValenzuela, Prat, Manzano Sanchez, \& Castaner, 2019) and Teacher Education (Blanco, 2015). The TPSR model has also been developed and implemented with different types of contexts, contents, ages and instructors, which suggests transference to a variety of settings (Hellison, 2003; Hellison \& Walsh, 2002), as well as in different countries, such as Ireland (Gordon \& Doyle, 2015); Portugal, Indonesia, Mexico and Spain (Martinek, 2009), New Zealand, Brazil, South Korea and Canada (Escartí, Wright, Pascual, \& Gutiérrez, 2015); Nepal, South Africa (Forneris, Whitley, \& Barker, 2013); China (Pan \& Keh, 2014); and East Timor (Baptista et al., 2016).

There has been an exponential and widespread increase in the implementation of TPSR-based interventions, as well as the associated body of literature (Metzler, 2005). A factor that has contributed to this growth was the creation of an official website called TPSR Alliance. "The Alliance website mentioned earlier has been developed with feedback from many attendees at our annual conferences" (Walsh \& Wright, 2016, p. 37). The first conference documents on the website (https://www.tpsr-alliance.org) in 2008: “The TPSR Alliance has been a space for such professional collaborations where members have been able to both benefit from and contribute to it by sharing research and practices revolving around developing responsible behaviors in youth using the teaching personal and social responsibility (TPSR) model" (Dunn, Hemphill, $\&$ Beaudoin, 2006). This website has been helpful not only for the scientific community, but also instructors (Wright, Whitley, \& Sabolboro, 2012) who aim to 


\section{Baptista, Corte-Real, Regueiras, Seo, Hemphill, Pereira, Dias, Martinek, Fonseca}

improve their pedagogical strategies (Escartí, Gutiérrez, Pascual, \& Wright, 2013) with the goal of helping their students care for themselves and for others in the future (Wright et al., 2012).

Currently, there are some literature reviews about TPSR model-based programs (Belando, FerrizMorell, \& Moreno-Murcia, 2012; Caballero-Blanco, Delgado-Noguera \& Escartí, 2013; Casey, 2014; Hellison \& Walsh, 2002; Pozo, Grao-Cruces \& PérezOrdás, 2016).

Hellison and Walsh (2002) conducted a literature review with the purpose of evaluating the impact of the Responsibility Model on underserved youngsters, since its inception. It included 26 studies, which were categorized by review process and publication status. Most studies (19) revealed the impact on participants' improvement within the program. Other studies (11) reported the impact on the transference from the programs to participants' lives outside the program.

Authors like Belando et al. (2012) and Casey (2014) conducted a review of the pedagogical models of intervention studies in the context of physical education, as well as other sports activities, which included, among other models, the model of Personal and Social Responsibility.

Recently, other review studies have been developed with only TPSR model-based programs, such as Caballero-Blanco et al. (2013), in which they compare the different studies that have implemented the TPSR model in the USA and in Spain, as well as the systematic review study of Pozo et al. (2016) on TPSR model-based programs within PE.

As reported earlier, there are some systematic reviews about the TPSR. However, none included the analysis of implementation exclusively in after-school settings.

In conclusion, for many physical educators who implemented the TPSR model in their sessions, this model is considered a viable and effective pedagogical approach in after-school contexts. This systematic review aims to analyze the studies included in peerreviewed journals mainly listed in a comprehensive list of over 200 publications trough TPSR Alliance within the after-school context.

\section{METHOD}

\section{Search strategy}

The question formulation is an important step to begin a systematic review and it should take into account relevant elements of the research design. For this study, a systematic interpretive review was drafted, focused mainly in qualitative work, to get a scientifichumanist perspectives, and results interpretation (Fernández-Ríos \& Buela-Casal, 2009), and three research questions were formulated: Which were the findings related to TPSR implementation in afterschool time settings? What research methodologies have been used to examine TPSR in after school time settings? What TPSR-related outcomes were experienced by students in after school time settings?

For the theoretical basis of the present study, articles retrieved from both the TPSR Alliance website and the PsycInfo database were assessed, as the Assessment of Multiple Systematic Reviews (Shea et al., 2007) postulates that at least two different sources should be used.

For the search from the TPSR Alliance website, the reference list updated in January of 2016 was used. This reference list contains Peer-Reviewed Academic and Professional Articles on TPSR and Other Publications Related to or Supporting TPSR. An additional search was conducted using PsycInfo, on April 13, 2017. The keywords used in the search systems of the database were: "teaching personal and social responsibility" OR "responsibility model" OR "personal and social responsibility program" AND "after school".

The term "after school" was defined because it is a broader term that includes extracurricular activities (such as activities organized and structured by school teachers) and other activities in or for the community, such as clubsand leadership programs, which are very common in school implementations of the TPSR model (Martinek, 2016).

The search was narrowed to articles between 2001 and 2016, with full text and peer review. Articles retrieved from the PsycInfo database during search procedures were exported to Endnote (electronic reference software) (Endnote x7, 2014). 


\section{Personal and social responsibility model systematic review}

The selection process of studies for eligibility and data extraction, as suggested by the Cochrane Collaboration (Figure 1) was undertaken by two independent researchers in order to avoid result bias during the selection process (Higgins \& Green, 2011). Any disagreements between researchers were discussed and a consensus was reached. Thus, the following selection criteria were applied a priori: a) using the TPSR model in the intervention/ impact on children and youngsters; b) peer-reviewed articles; c) the articles must include participants of TPSR model interventions in after-school contexts; d) articles published between 2001 and January 2016. The exclusion criteria were: a) intervention conducted in a physical education context; b) no access to the full text; c) grey literature (thesis, book, book chapters), as this type of literature is not submitted for peer-review; d) articles that only described strategies of TPSR in after-school contexts.

The degree of agreement was calculated by Cohen's Kappa .83, p< 001 (Siegel \& Castellan, 1988), exhibiting an almost perfect agreement (Landis \& Koch, 1977).

Figure 1

Flow diagram of literature search

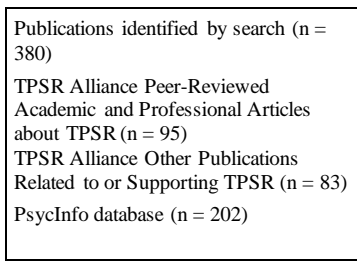

Full text articles reviewed $(n=57)$

Full text articles included in review $(\mathrm{n}=27)$

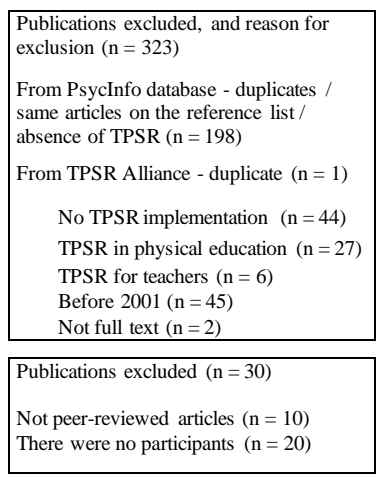

Not peer-reviewed articles $(\mathrm{n}=10)$
There were no participants $(\mathrm{n}=20)$

\section{Score of studies}

The criteria for the measured score of the 27 papers included studies adapted from the Strengthening the Reporting of Observational Studies in Epidemiology (STROBE) statement (Vandenbroucke et al., 2007) and the Consolidated Standards of Reporting Trials (CONSORT) statement (Moher, Schulz \& Altman, 2001).
Question 1: Did the study provide a detailed description of the program implementation? ' 0 ' not included; ' 1 'description included but it is brief and imprecise (e.g., format plan, duration, included information about participants but did not report details about activities); '2' detailed description of the activities was included.

Question 2: Did the study report the duration of the intervention? ' 0 ' it was not reported; ' 1 ' nine or less sessions; ' 2 ' between 10 and 12 or more sessions.

Question 3: Did the paper report validity and reliability. ' 0 ' not reported; ' 1 ' in quantitative studies was shown the validity and/ or reliability of the instruments. In qualitative studies was shown the validity through at least one type of triangulation (triangulation of methods, sources, triangulation of the researchers, and triangulation of the theory/perspective). ' 2 ' in quantitative studies described the steps to the validity and reliability supported in the literature. In qualitative studies was shown the validity through some sort of triangulation with the detailed description of the process of triangulation.

Question 4: Did the study report methodological design and analysis? ' 0 ' not reported; ' 1 ' in quantitative studies was shown the methods (questionnaire, direct observation), design (pre- and post-test) and the type of statistics used. In qualitative studies was shown the methods (interviews, focus group, case study, observation, documents) used in the study; ' 2 ' in quantitative studies was made a detailed description of the methods (description of the instrument), described the purpose of the application of statistics used in the study. In qualitative studies have been made description of the units of analysis (participants, groups), and a description of the category approach (deductive and inductive).

Question 5: Did the paper report findings? ' 0 ' no findings reported; ' 1 ' reported findings from authors but did not report findings from all data sources; ' 2 ' reported findings from authors and all other data sources (e.g., from interviews and questionnaires).

All questions were rated from ' 0 ' to ' 2 ', depending on the criteria, shown below in Table 1 . The maximum score is 10 points. 


\section{Baptista, Corte-Real, Regueiras, Seo, Hemphill, Pereira, Dias, Martinek, Fonseca}

Table 1

List of included studies with scores

\begin{tabular}{|c|c|c|c|c|c|c|}
\hline Authors/ Date & $\begin{array}{l}\text { Q. } \\
1\end{array}$ & $\begin{array}{l}\text { Q. } \\
2\end{array}$ & Q. 3 & $\begin{array}{l}\mathrm{Q} . \\
4\end{array}$ & $\begin{array}{l}\mathrm{Q} . \\
5\end{array}$ & $\begin{array}{l}\text { Total } \\
\text { Score }\end{array}$ \\
\hline Beale (2012) & 2 & 2 & 0 & 1 & 2 & 7 \\
\hline Bean and Forneris (2015) & 2 & 2 & 2 & 2 & 2 & 10 \\
\hline Buchanan (2001) & 2 & 1 & 2 & 2 & 2 & 9 \\
\hline Buckle and Walsh (2013) & 2 & 2 & 0 & 0 & 1 & 5 \\
\hline $\begin{array}{l}\text { Coulson, Irwin, and Wright } \\
\text { (2012) }\end{array}$ & 1 & 0 & 0 & 1 & 1 & 3 \\
\hline $\begin{array}{l}\text { Hayden, Baltzell, Kilty, and } \\
\text { McCarthy (2012) }\end{array}$ & 2 & 2 & 1 & 2 & 2 & 9 \\
\hline Hellison and Wright (2003) & 2 & 2 & 2 & 2 & 2 & 10 \\
\hline Lee and Martinek (2012) & 1 & 1 & 2 & 1 & 1 & 6 \\
\hline $\begin{array}{l}\text { Martinek and Schilling } \\
\text { (2003) }\end{array}$ & 2 & 2 & 0 & 1 & 1 & 6 \\
\hline $\begin{array}{l}\text { Martinek, Schiling, and } \\
\text { Hellison (2006) }\end{array}$ & 1 & - & 0 & 1 & 1 & 3 \\
\hline $\begin{array}{l}\text { Martinek, Schiling, and } \\
\text { Johnson (2001) }\end{array}$ & 2 & 2 & 0 & 2 & 2 & 8 \\
\hline Schilling (2001) & 1 & 2 & 2 & 2 & 2 & 9 \\
\hline Walsh (2007) & 2 & 2 & 0 & 1 & 1 & 6 \\
\hline Walsh (2008) & 2 & 1 & 1 & 1 & 1 & 6 \\
\hline $\begin{array}{l}\text { Walsh, Ozaeta, and Wright } \\
\text { (2010) }\end{array}$ & 2 & 2 & 2 & 2 & 2 & 10 \\
\hline $\begin{array}{l}\text { Wright, Dyson, and Moten } \\
\text { (2012) }\end{array}$ & 1 & 2 & 1 & 1 & 1 & 6 \\
\hline Wright, et al., (2012) & 2 & 1 & 0 & 0 & 1 & 4 \\
\hline Wright (2012) & 2 & 2 & 0 & 1 & 0 & 5 \\
\hline $\begin{array}{l}\text { Wright, White and Gaebier- } \\
\text { Spira (2004) }\end{array}$ & 2 & 2 & 1 & 2 & 2 & 9 \\
\hline Whitley (2011) & 2 & 1 & 0 & 0 & 1 & 4 \\
\hline $\begin{array}{l}\text { Hammond-Diedrich and } \\
\text { Walsh (2006) }\end{array}$ & 2 & 1 & 1 & 2 & 2 & 8 \\
\hline Schilling (2007) & 1 & 0 & 0 & 2 & 2 & 5 \\
\hline Lee and Martinek (2009) & 1 & 1 & 1 & 2 & 2 & 7 \\
\hline Forneris et al. (2013) & 1 & 0 & 0 & 0 & 1 & 2 \\
\hline $\begin{array}{l}\text { Newton, Watson, Kim, and } \\
\text { Beacham (2006) }\end{array}$ & 2 & 1 & 0 & 2 & 2 & 7 \\
\hline $\begin{array}{l}\text { Bean, Kendellen, and } \\
\text { Forneris (2016) }\end{array}$ & 2 & 2 & 0 & 2 & 2 & 8 \\
\hline Whitley and Gould (2011) & 2 & 1 & 0 & 0 & 1 & 4 \\
\hline
\end{tabular}

\section{RESULTS}

The results will be presented in Table 2, which includes information regarding the authors and year, focus, local and duration of the program, context and physical activity, participants in the program, study sample, control group, study type, measurement instrument, design, data analysis, and results. They will also be described in three sections, according to the research questions.
Which were the findings related to TPSR implementation in after school time settings?

\section{Duration of the program}

At least nine activity sessions are required for intervention in order to improve and facilitate the transfer (Catalano, Berglung, Ryan, Lonczak, \& Hawkins, 2004; Petitpas, Cornelius, Raalte, \& Jones, 2005). In the present study it was found that most of the interventions 15/27 fulfilled this assumption (Beale, 2012; Bean \& Forneris, 2015; Buckle \& Walsh, 2013; Forneris et al., 2013; Hayden et al., 2012; Hellison \& Wright, 2003; Martinek et al., 2001; Martinek \& Schilling, 2003; Schilling, 2001; Walsh, 2007, 2008; Walsh et al., 2010; Wright et al., 2012; Wright et al., 2012; Wright \& Gaebler-Spira, 2004). Still, there were $8 / 27$ who performed interventions under nine sessions (Buchanan, 2001; HammondDiedrich \& Walsh, 2006; Lee \& Martinek, 2009, 2012; Newton et al., 2006; Whitley, 2011; Whitley \& Gould, 2011; Wright, 2012). However, only Whitley (2011) reported the five sessions of intervention were not sufficient to provide constructive criticism or to make an impact on the welfare (Buchanan, 2001).

Some studies (3/27) did not report the duration of the intervention (Coulson et al., 2012; Martinek et al., 2006; Schilling, 2007).

\section{Intervention location}

Most after-school interventions based on the TPSR model (25/27) were conducted in the United States of America (Bean et al., 2015; Beale, 2012; Buchanan, 2001; Buckle \& Walsh, 2013; Coulson et al., 2012; Forneris et al. 2013; Hammond-Diedrich \& Walsh, 2006; Hayden et al., 2012; Hellison \& Wright, 2003; Lee \& Martinek, 2012; Lee \& Martinek, 2009; Martinek \& Schilling, 2003; Martinek et al., 2006; Martinek et al., 2001; Newton et al., 2006; Schilling, 2001; Schilling, 2007; Walsh, 2007, 2008; Walsh et al., 2010; Whitley \& Gould, 2011; Wright, 2012; Wright et al., 2012; Wright et al., 2012; Wright et al., 2004).

However, it was possible to find few interventions with the TPSR model in other developed countries such as Canada, Nepal and South Africa (Bean \& Forneris, 2015; Forneris et al., 2013; Whitley, 2011). 
Personal and social responsibility model systematic review

Table 2 General overview of the literature review

\begin{tabular}{|c|c|c|c|c|c|c|c|c|c|c|c|}
\hline \multirow[b]{2}{*}{$\begin{array}{l}\text { Author(s)/ } \\
\text { year }\end{array}$} & \multirow[b]{2}{*}{ Focus } & \multirow[b]{2}{*}{$\begin{array}{l}\text { Local/ } \\
\text { Duration } \\
\text { of the } \\
\text { program }\end{array}$} & \multirow[b]{2}{*}{$\begin{array}{l}\text { Context/ } \\
\text { Physical } \\
\text { activity }\end{array}$} & \multirow[b]{2}{*}{$\begin{array}{l}\text { Participants in } \\
\text { the program }\end{array}$} & \multicolumn{6}{|c|}{ Methodological design } & \multirow[b]{2}{*}{ Results } \\
\hline & & & & & $\begin{array}{c}\text { Study } \\
\text { sample }\end{array}$ & $\begin{array}{l}\text { Control } \\
\text { group }\end{array}$ & Study type & $\begin{array}{c}\text { Measurement } \\
\text { instrument }\end{array}$ & $\begin{array}{l}\text { Design } \\
\text { (pre/ } \\
\text { post } \\
\text { test) }\end{array}$ & $\begin{array}{c}\text { Data } \\
\text { analysis }\end{array}$ & \\
\hline $\begin{array}{l}\text { Beale } \\
(2012)\end{array}$ & $\begin{array}{l}\text { Program was designed } \\
\text { in the shape of } \\
\text { lifeguards' course } \\
\text { considering the skills } \\
\text { and certification } \\
\text { acquired during the } \\
\text { course. }\end{array}$ & $\begin{array}{l}\text { USA } \\
192 \\
\text { sessions }\end{array}$ & $\begin{array}{l}\text { Lifeguards' } \\
\text { course } \\
\text { Swimming } \\
\text { and } \\
\text { lifesaving } \\
\text { skills }\end{array}$ & $\begin{array}{l}\mathrm{N}=300 \\
\text { Female } \\
\text { Male } \\
16 \text { years old } \\
\text { and older }\end{array}$ & $\begin{array}{l}\mathrm{N}=300 \\
\text { Female } \\
\text { Male } \\
16 \text { years } \\
\text { old and } \\
\text { older }\end{array}$ & No & $\begin{array}{l}\text { Qualitative } \\
\text { assessment }\end{array}$ & $\begin{array}{l}\text { Interview, } \\
\text { Documents, } \\
\text { Field notes } \\
\text { Observations }\end{array}$ & No & $\begin{array}{l}\text { Not } \\
\text { reported }\end{array}$ & $\begin{array}{l}\text { Students became more water safe, earned one or } \\
\text { more American Red Cross instructional swimming } \\
\text { certificate, and demonstrated that the program } \\
\text { aided them inside and outside the pool. }\end{array}$ \\
\hline $\begin{array}{l}\text { Bean and } \\
\text { Forneris } \\
(2015)\end{array}$ & $\begin{array}{l}\text { Examined how well the } \\
\text { implementation of the } \\
\text { program Girls Just } \\
\text { Wanna Have Fun } \\
\text { (GJWHF) followed the } \\
\text { five levels of the TPSR } \\
\text { model, using a time } \\
\text { series analysis. }\end{array}$ & $\begin{array}{l}\text { Canada } \\
30 \\
\text { sessions }\end{array}$ & $\begin{array}{l}\text { Summer } \\
\text { camp, } \\
\text { Fitness, } \\
\text { Team sports }\end{array}$ & $\begin{array}{l}\mathrm{N}=12 \text { Female } \\
11-14 \text { years } \\
\text { old } \\
\mathrm{N}=5 \text { staff, } \\
\text { Female } \\
\text { Male } \\
28.6 \text { years old }\end{array}$ & $\begin{array}{l}\mathrm{N}=12 \\
\text { Female } \\
11-14 \\
\text { years old } \\
\mathrm{N}=5 \\
\text { staff, } \\
\text { Female } \\
\text { Male } \\
28.6 \\
\text { years old }\end{array}$ & No & $\begin{array}{l}\text { Quantitative } \\
\text { assessment }\end{array}$ & $\begin{array}{l}\text { Personal and } \\
\text { Social } \\
\text { Responsibility } \\
\text { Questionnaire } \\
\text { (PSRQ), } \\
\text { Leader } \\
\text { logbook, } \\
\text { Group } \\
\text { evaluation }\end{array}$ & Yes & $\begin{array}{l}\text { Descriptive } \\
\text { statistics } \\
\text { Dependent } \\
\text { T-Test } \\
\text { Time series } \\
\text { analysis } \\
\text { Cronbach's } \\
\text { Alpha }\end{array}$ & $\begin{array}{l}\text { PSRQ results of the } 12 \text { girls showed an increase in } \\
\text { the social responsibility of pre }(\mathrm{M}=4.60 ; \mathrm{SD}=94) \\
\text { to post }(\mathrm{M}=5.13 ; \mathrm{SD}=.58) \text {, although this } \\
\text { difference approached significance only, } \mathrm{t}(7)=- \\
2.17, \mathrm{p}=.066 \text {. On the other hand, there was a } \\
\text { slight lack of personal responsibility of pre }(\mathrm{M}= \\
4.66 ; \mathrm{SD}=1.09) \text { to post }(\mathrm{M}=4.41 ; \mathrm{SD}=1.12), \\
\text { however, was not significant, } \mathrm{t}(7)=.657, \mathrm{p}=.532 \text {. } \\
\text { The staff perceived the } 12 \text { girls had improved in } \\
\text { self-control }(2.0 \text { and } 3.0) \text {, self-coaching }(3.0 \text { to } 4.0) \\
\text { and leadership ( } 2.0 \text { to } 4.0) \text {. }\end{array}$ \\
\hline $\begin{array}{l}\text { Buchanan } \\
(2001)\end{array}$ & $\begin{array}{l}\text { Examined the } \\
\text { implementation of } \\
\text { Hellison responsibility } \\
\text { model (TPSR) by staff } \\
\text { at an instructional sports } \\
\text { camp for at-risk } \\
\text { youngsters. }\end{array}$ & $\begin{array}{l}\text { USA } \\
5 \\
\text { sessions }\end{array}$ & $\begin{array}{l}\begin{array}{l}\text { Summer } \\
\text { camp }\end{array} \\
\text { Fitness }\end{array}$ & $\begin{array}{l}\mathrm{N}=200 \\
\text { youngsters } \\
12-13 \text { years } \\
\text { old } \\
\mathrm{N}=6 \text { staff }(3 \\
\text { Female and } 3 \\
\text { Male) }\end{array}$ & $\begin{array}{l}\mathrm{N}=6 \\
\text { staff }(3 \\
\text { Female } \\
\text { and } 3 \\
\text { Male) }\end{array}$ & No & $\begin{array}{l}\text { Qualitative } \\
\text { assessment }\end{array}$ & $\begin{array}{l}\text { Interview, } \\
\text { Observation } \\
\text { Journal }\end{array}$ & No & $\begin{array}{l}\text { Naturalistic } \\
\text { data } \\
\text { Emergent } \\
\text { themes }\end{array}$ & $\begin{array}{l}\text { The camp duration was too short and the model } \\
\text { was not entirely implemented as a vehicle for well- } \\
\text { being. While some staff members modeled the } \\
\text { qualities that they demanded of the youngsters, } \\
\text { others displayed inflexibility, authoritarianism, and } \\
\text { disrespect. Strategies such as teachable moments, } \\
\text { feedback, and reflection were used to facilitate } \\
\text { self-awareness and empowerment. }\end{array}$ \\
\hline
\end{tabular}

\section{4 \\ ODsicología}




\section{Baptista, Corte-Real, Regueiras, Seo, Hemphill, Pereira, Dias, Martinek, Fonseca}

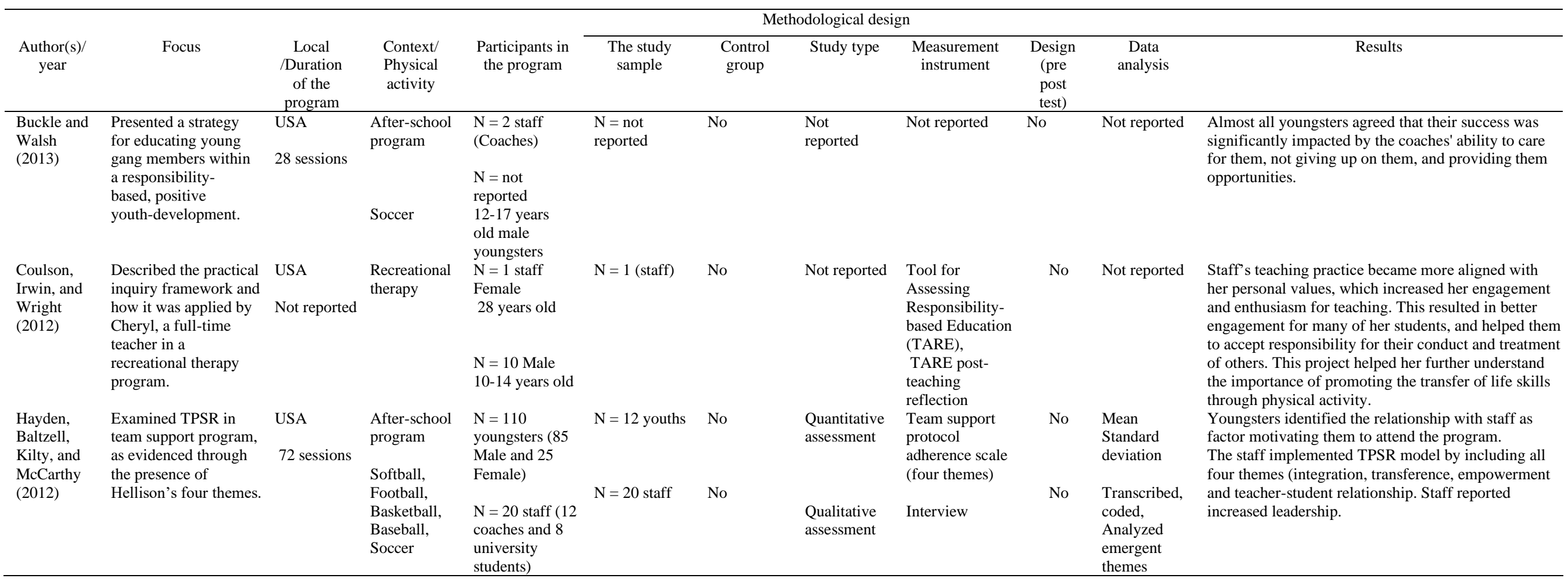


Personal and social responsibility model systematic review

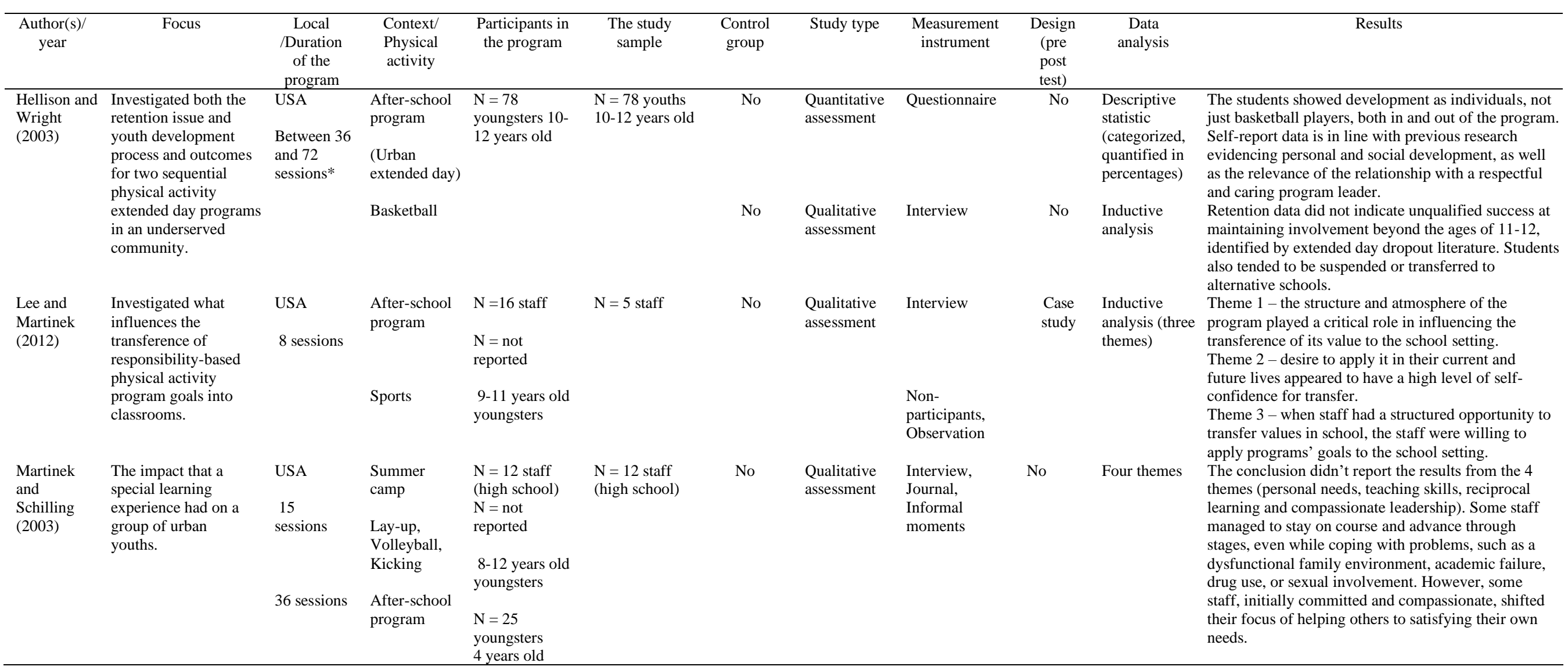

\section{Rnome \\ - Psicoamericana de \\ Veporte}


Baptista, Corte-Real, Regueiras, Seo, Hemphill, Pereira, Dias, Martinek, Fonseca

\begin{tabular}{|c|c|c|c|c|c|c|c|c|c|c|c|}
\hline \multirow[b]{2}{*}{$\begin{array}{c}\text { Author(s)/ } \\
\text { year }\end{array}$} & \multirow[b]{2}{*}{ Focus } & \multirow[b]{2}{*}{$\begin{array}{l}\text { Local } \\
\text { /Duration } \\
\text { of the } \\
\text { program }\end{array}$} & \multirow[b]{2}{*}{$\begin{array}{l}\text { Context/ } \\
\text { Physical } \\
\text { activity }\end{array}$} & \multirow[b]{2}{*}{$\begin{array}{l}\text { Participants in } \\
\text { the program }\end{array}$} & \multicolumn{5}{|c|}{ Methodological design } & \multirow[b]{2}{*}{$\begin{array}{l}\text { Data } \\
\text { analysis }\end{array}$} & \multirow[b]{2}{*}{ Results } \\
\hline & & & & & $\begin{array}{l}\text { The study } \\
\text { sample }\end{array}$ & $\begin{array}{l}\text { Control } \\
\text { group }\end{array}$ & Study type & $\begin{array}{c}\text { Measurement } \\
\text { instrument }\end{array}$ & $\begin{array}{l}\text { Design } \\
\text { (pre } \\
\text { post } \\
\text { test) } \\
\end{array}$ & & \\
\hline $\begin{array}{l}\text { Martinek } \\
\text { et al. } \\
\text { (2006) }\end{array}$ & $\begin{array}{l}\text { Description how } \\
\text { youth leadership } \\
\text { evolved in two } \\
\text { education programs. }\end{array}$ & $\begin{array}{l}\text { USA } \\
\text { Not } \\
\text { reported }\end{array}$ & $\begin{array}{l}\text { After- } \\
\text { school } \\
\text { program }\end{array}$ & $\begin{array}{l}\mathrm{N}=4 \text { staff } \\
14-19 \text { years } \\
\text { old }\end{array}$ & $\begin{array}{l}\mathrm{N}=4 \text { staff } \\
14-19 \text { years } \\
\text { old }\end{array}$ & No & $\begin{array}{l}\text { Quantitative } \\
\text { assessment }\end{array}$ & $\begin{array}{l}\text { Interview, } \\
\text { Reflection, } \\
\text { Field notes } \\
\text { Questionnaire }\end{array}$ & No & Not reported & $\begin{array}{l}\text { The stages were exemplified by four case studies, } \\
\text { which evidenced the evolution of adolescents from } \\
\text { self-serving participants to caring and compassionate } \\
\text { staff. Although some regression was occasionally } \\
\text { displayed by staff, they also shifted beyond their } \\
\text { present stage to an advanced stage. Their ease in } \\
\text { extending their leadership and compassion to } \\
\text { younger participants was strongly influenced by their } \\
\text { personal lives. }\end{array}$ \\
\hline $\begin{array}{l}\text { Martinek } \\
\text { et al. } \\
\text { (2001) }\end{array}$ & $\begin{array}{l}\text { The impact of a } 6 \text { - } \\
\text { month sport club and } \\
\text { mentoring program. }\end{array}$ & $\begin{array}{l}\text { USA } \\
24 \text { sessions }\end{array}$ & $\begin{array}{l}\text { After-school } \\
\text { program }\end{array}$ & $\begin{array}{l}\mathrm{N}=8 \text { staff } \\
\mathrm{N}=16 \\
\text { youngsters }\end{array}$ & $\begin{array}{l}\mathrm{N}=16 \\
\text { youths }\end{array}$ & No & $\begin{array}{l}\text { Qualitative } \\
\text { assessment }\end{array}$ & $\begin{array}{l}\text { Interview, } \\
\text { Journal entries } \\
\text { Journal cards }\end{array}$ & Post & 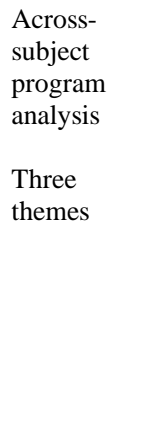 & $\begin{array}{l}\text { Personal responsibility: } 88 \% \text { of youngsters seemed } \\
\text { to show effort most or some of the time; } 37 \% \\
\text { youngsters tried to set goals either most of the time, } \\
\text { or some of the time, whereas the others, } 63 \% \text {, set } \\
\text { them little of the time. Social responsibility: } 63 \% \text { of } \\
\text { the youngsters were capable of showing respect and } \\
\text { self-control most or some of the time in the } \\
\text { classroom. Conversely, } 37 \% \text { did this little of the time } \\
\text { and often got into trouble with the teacher or } \\
\text { principal; } 50 \% \text { of youngsters were caring towards } \\
\text { others most or some of the time. Transference: } 62 \% \\
\text { of youngsters evidenced medium and high levels of } \\
\text { transference. }\end{array}$ \\
\hline $\begin{array}{l}\text { Schilling } \\
\text { (2001) }\end{array}$ & $\begin{array}{l}\text { Examined } \\
\text { underserved youngs } \\
\text { participants' } \\
\text { perceptions of } \\
\text { commitment to an } \\
\text { extended day } \\
\text { physical activity } \\
\text { program. }\end{array}$ & $\begin{array}{l}\text { USA } \\
16 \text { sessions }\end{array}$ & $\begin{array}{l}\text { After-school } \\
\text { program }\end{array}$ & $\begin{array}{l}\mathrm{N}=7 \\
\text { youngsters } \\
12-15 \text { years } \\
\text { old }\end{array}$ & $\mathrm{N}=7$ youths & No & $\begin{array}{l}\text { Qualitative } \\
\text { assessment }\end{array}$ & Interview & No & $\begin{array}{l}\text { Inductive } \\
\text { analysis } \\
\text { (two } \\
\text { themes) }\end{array}$ & $\begin{array}{l}\text { Theme } 1 \text { - antecedents to commitment: young } \\
\text { participants reported that program commitment was } \\
\text { positively influenced by relationships with peers and } \\
\text { staff, as well as type of activity, although the specific } \\
\text { activity could also serve as an obstacle. } \\
\text { Theme } 2 \text { - nature of commitment: effort and } \\
\text { persistence were reflective of program commitment. } \\
\text { Most participants also mentioned that having fun and } \\
\text { enjoying the program reflected their commitment to } \\
\text { it. }\end{array}$ \\
\hline
\end{tabular}


Personal and social responsibility model systematic review

\begin{tabular}{|c|c|c|c|c|c|c|c|c|c|c|c|}
\hline \multirow[b]{2}{*}{$\begin{array}{c}\text { Author(s)/ } \\
\text { year }\end{array}$} & \multirow[b]{2}{*}{ Focus } & \multirow[b]{2}{*}{$\begin{array}{l}\text { Local } \\
\text { /Duration } \\
\text { of the } \\
\text { program }\end{array}$} & \multirow[b]{2}{*}{$\begin{array}{c}\text { Context/ } \\
\text { Physical } \\
\text { activity }\end{array}$} & \multirow[b]{2}{*}{$\begin{array}{l}\text { Participants in } \\
\text { the program }\end{array}$} & \multicolumn{5}{|c|}{ Methodological design } & \multirow[b]{2}{*}{$\begin{array}{l}\text { Data } \\
\text { analysis }\end{array}$} & \multirow[b]{2}{*}{ Results } \\
\hline & & & & & $\begin{array}{l}\text { The study } \\
\text { sample }\end{array}$ & $\begin{array}{l}\text { Control } \\
\text { group }\end{array}$ & Study type & $\begin{array}{c}\text { Measurement } \\
\text { instrument }\end{array}$ & $\begin{array}{l}\text { Design } \\
\text { (pre } \\
\text { post } \\
\text { test) }\end{array}$ & & \\
\hline $\begin{array}{l}\text { Walsh } \\
(2008)\end{array}$ & $\begin{array}{l}\text { Examined the } \\
\text { combination of TPSR } \\
\text { with the theory of } \\
\text { possible selves. }\end{array}$ & $\begin{array}{l}\text { USA } \\
9 \text { sessions }\end{array}$ & $\begin{array}{l}\text { After-school } \\
\text { program }\end{array}$ & $\begin{array}{l}\mathrm{N}=12 \text { staff } \\
(7 \text { Female and } \\
5 \text { Male) } \\
13-14 \text { years } \\
\text { old } \\
\mathrm{N}=20 \\
\text { youngsters } 10 \\
\text { years old }\end{array}$ & $\mathrm{N}=12$ staff & No & $\begin{array}{l}\text { Qualitative } \\
\text { assessment } \\
\text { Quantitative } \\
\text { assessment }\end{array}$ & $\begin{array}{l}\begin{array}{l}\text { Document } \\
\text { analysis, } \\
\text { interviews }\end{array} \\
\text { Lesson } \\
\text { observations, } \\
\text { observational } \\
\text { field notes }\end{array}$ & No & $\begin{array}{l}\text { Case study, } \\
\text { Inductive } \\
\text { analysis, } \\
\text { Not reported }\end{array}$ & $\begin{array}{l}\text { The results supported reflection related to possible } \\
\text { futures. It was difficult to control and distinguish the } \\
\text { impact of the regular TPSR program from added } \\
\text { value of experiencing a career and the impact of the } \\
\text { possible-selves theory. }\end{array}$ \\
\hline $\begin{array}{l}\text { Walsh et } \\
\text { al. (2010) }\end{array}$ & $\begin{array}{l}\text { Examined the degree } \\
\text { of transference of the } \\
\text { four primary TPSR } \\
\text { goals from a } \\
\text { Coaching Club } \\
\text { program to the } \\
\text { participants' school } \\
\text { environment. }\end{array}$ & $\begin{array}{l}\text { USA } \\
45 \text { sessions }\end{array}$ & $\begin{array}{l}\text { After-school } \\
\text { program }\end{array}$ & $\begin{array}{l}\mathrm{N}=13 \\
\text { youngsters } 9- \\
11 \text { years old } \\
\mathrm{N}=3 \text { staff }\end{array}$ & $\begin{array}{l}\mathrm{N}=13 \\
\text { youngsters } 9 \\
11 \text { years old } \\
\mathrm{N}=3 \text { staff }\end{array}$ & No & $\begin{array}{l}\text { Qualitative } \\
\text { assessment }\end{array}$ & $\begin{array}{l}\text { Interviews } \\
\text { observations } \\
\text { documents }\end{array}$ & No & $\begin{array}{l}\text { Case study, } \\
\text { Inductive } \\
\text { and } \\
\text { deductive } \\
\text { analysis }\end{array}$ & $\begin{array}{l}\text { All young people revealed a greater understanding, } \\
\text { growth and impact on transfer of respect the rights } \\
\text { and feelings of others (level 1), } 10 \text { youngsters } \\
\text { reported the transfer of their desire to work more and } \\
\text { work together (level 2), as well as, self-employment } \\
\text { and create goals (level 3), } 11 \text { youngsters reported the } \\
\text { transference towards to help others and leadership } \\
\text { (level 4) for the school environment. The three staff } \\
\text { members confirmed with examples the levels in } \\
\text { school environment. }\end{array}$ \\
\hline
\end{tabular}

\section{$8=$}


Baptista, Corte-Real, Regueiras, Seo, Hemphill, Pereira, Dias, Martinek, Fonseca

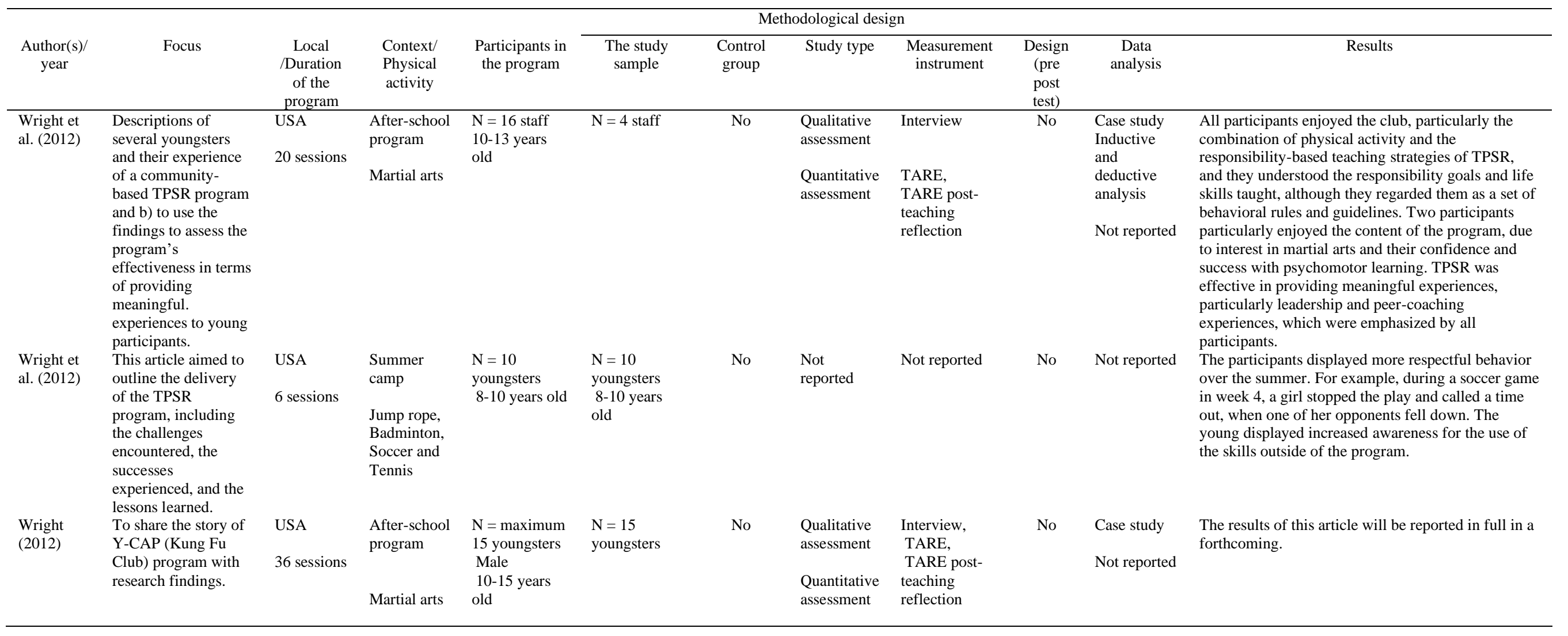


Personal and social responsibility model systematic review

\begin{tabular}{|c|c|c|c|c|c|c|c|c|c|c|c|}
\hline \multirow[b]{2}{*}{$\begin{array}{c}\text { Author(s)/ } \\
\text { year }\end{array}$} & \multirow[b]{2}{*}{ Focus } & \multirow[b]{2}{*}{$\begin{array}{l}\text { Local } \\
\text { /Duration } \\
\text { of the } \\
\text { program }\end{array}$} & \multirow[b]{2}{*}{$\begin{array}{l}\text { Context/ } \\
\text { Physical } \\
\text { activity }\end{array}$} & \multirow[b]{2}{*}{$\begin{array}{l}\text { Participants in } \\
\text { the program }\end{array}$} & \multicolumn{5}{|c|}{ Methodological design } & \multirow[b]{2}{*}{$\begin{array}{l}\text { Data } \\
\text { analysis }\end{array}$} & \multirow[b]{2}{*}{ Results } \\
\hline & & & & & $\begin{array}{l}\text { The study } \\
\text { sample }\end{array}$ & $\begin{array}{l}\text { Control } \\
\text { group }\end{array}$ & Study type & $\begin{array}{c}\text { Measurement } \\
\text { instrument }\end{array}$ & $\begin{array}{l}\text { Design } \\
\text { (pre } \\
\text { post } \\
\text { test) } \\
\end{array}$ & & \\
\hline $\begin{array}{l}\text { Whitley } \\
\text { (2011) }\end{array}$ & $\begin{array}{l}\text { Critical elements for } \\
\text { program success and } \\
\text { the challenges that } \\
\text { were faced during the } \\
\text { design and } \\
\text { implementation } \\
\text { phases of the } \\
\text { program. }\end{array}$ & $\begin{array}{l}\text { South } \\
\text { Africa } \\
5 \text { sessions }\end{array}$ & $\begin{array}{l}\text { After-school } \\
\text { program }\end{array}$ & $\begin{array}{l}\mathrm{N}=20 \text { to } 35 \\
\text { youngsters } \\
6-14 \text { years old }\end{array}$ & $\begin{array}{l}\mathrm{N}=20 \text { to } 35 \\
\text { youngsters }\end{array}$ & No & $\begin{array}{l}\text { Not } \\
\text { reported }\end{array}$ & Not reported & No & Not reported & $\begin{array}{l}\text { Five sessions were just not enough time for the } \\
\text { facilitators to understand the model and provide } \\
\text { constructive criticism. There also have been } \\
\text { problems of communication since the language used } \\
\text { was not the native language and had to use a } \\
\text { translator. }\end{array}$ \\
\hline $\begin{array}{l}\text { Hammond- } \\
\text { Diedrich } \\
\text { and Walsh } \\
(2006)\end{array}$ & $\begin{array}{l}\text { The impact of } \\
\text { Responsibility Model } \\
\text { (RM)-based, cross- } \\
\text { age teaching program } \\
\text { that brought together } \\
\text { selected urban youth } \\
\text { from different RM } \\
\text { programs. }\end{array}$ & $\begin{array}{l}\text { USA } \\
8 \text { sessions }\end{array}$ & $\begin{array}{l}\text { Summer } \\
\text { sport } \\
\text { program }\end{array}$ & $\begin{array}{l}\mathrm{N}=8 \text { staff } \\
\text { Male } \\
11-15 \text { years } \\
\text { old } \\
\mathrm{N}=40 \\
\text { youngsters }\end{array}$ & $\mathrm{N}=8$ staff & No & $\begin{array}{l}\text { Qualitative } \\
\text { assessment }\end{array}$ & $\begin{array}{l}\text { Interview } \\
\text { Field notes } \\
\text { Lesson } \\
\text { observations }\end{array}$ & No & $\begin{array}{l}\text { Case study } \\
\text { Inductive } \\
\text { analysis }\end{array}$ & $\begin{array}{l}\text { Staff improved their coaching skills and viewed } \\
\text { themselves as effective coaches who positively } \\
\text { impacted the fourth graders. Staff also enjoyed the } \\
\text { time spent at the university and considered enrolling } \\
\text { in one in the future. Staff believed the program } \\
\text { would aid their academic performance. }\end{array}$ \\
\hline
\end{tabular}

\section{Sos uciedad \\ 7 Iberoamericana de \\ ODsicología}


Baptista, Corte-Real, Regueiras, Seo, Hemphill, Pereira, Dias, Martinek, Fonseca

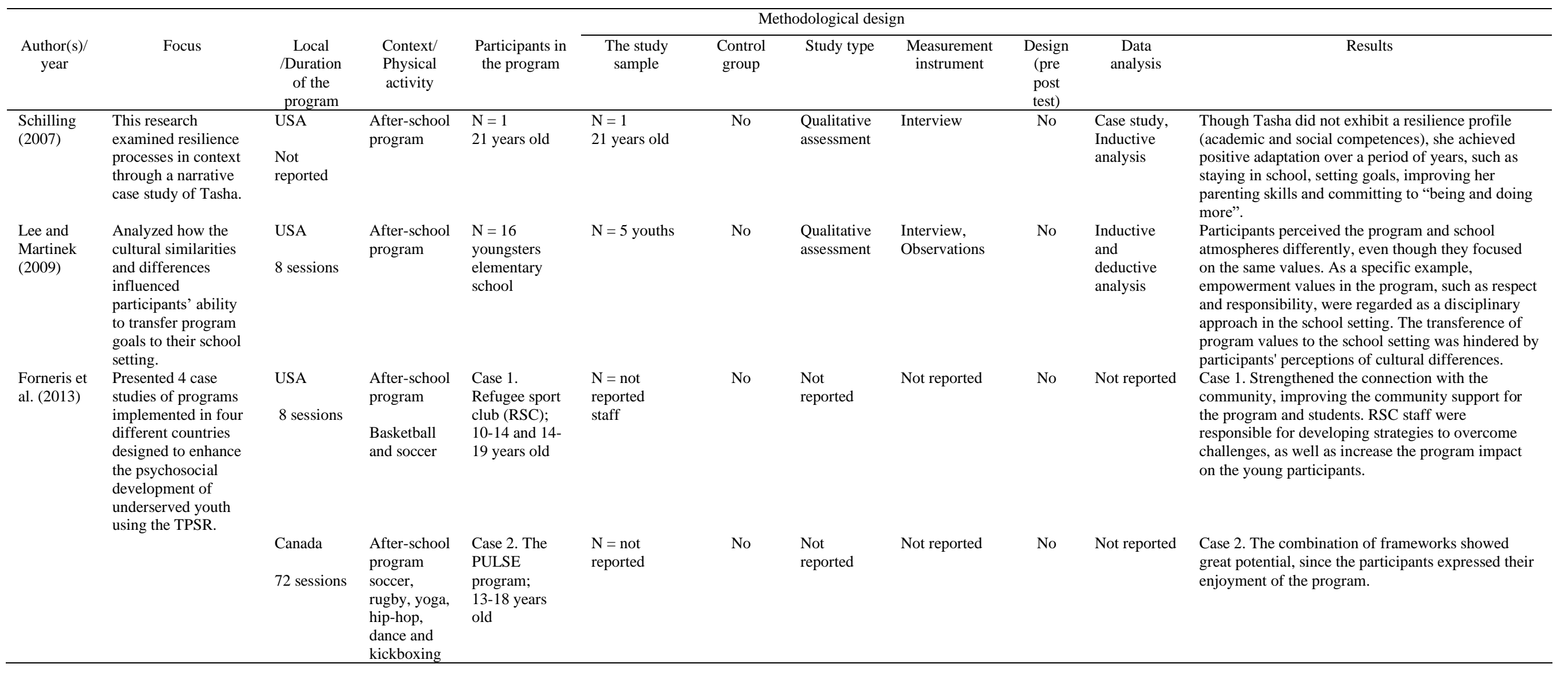


Personal and social responsibility model systematic review

\begin{tabular}{|c|c|c|c|c|c|c|c|c|c|c|c|}
\hline \multirow[b]{2}{*}{$\begin{array}{l}\text { Author(s) } \\
\text { / year }\end{array}$} & \multirow[b]{2}{*}{ Focus } & \multirow[b]{2}{*}{$\begin{array}{l}\text { Local } \\
\text { /Duration } \\
\text { of the } \\
\text { program }\end{array}$} & \multirow[b]{2}{*}{$\begin{array}{l}\text { Context/ } \\
\text { Physical } \\
\text { activity }\end{array}$} & \multirow[b]{2}{*}{$\begin{array}{l}\text { Participants } \\
\text { in the } \\
\text { program }\end{array}$} & \multicolumn{5}{|c|}{ Methodological design } & \multirow[b]{2}{*}{$\begin{array}{c}\text { Data } \\
\text { analysis }\end{array}$} & \multirow[b]{2}{*}{ Results } \\
\hline & & & & & $\begin{array}{c}\text { The study } \\
\text { sample }\end{array}$ & $\begin{array}{l}\text { Control } \\
\text { group }\end{array}$ & Study type & $\begin{array}{l}\text { Measurement } \\
\text { instrument }\end{array}$ & $\begin{array}{l}\text { Design } \\
\text { (pre } \\
\text { post } \\
\text { test) } \\
\end{array}$ & & \\
\hline $\begin{array}{l}\text { Newton } \\
\text { et al. } \\
\text { (2006) }\end{array}$ & $\begin{array}{l}\text { Examined the } \\
\text { relationship between } \\
\text { constructs of } \\
\text { achievement goal theory } \\
\text { and } \\
\text { indices of positive sport } \\
\text { participation in a } \\
\text { noncompulsory physical } \\
\text { activity setting with } \\
\text { underserved youth in } \\
\text { NYSP program. }\end{array}$ & $\begin{array}{l}\text { USA } \\
5 \\
\text { sessions }\end{array}$ & $\begin{array}{l}\text { Summer } \\
\text { camp } \\
\text { Golf, } \\
\text { Tennis, } \\
\text { Swimming } \\
\text { and Soccer }\end{array}$ & $\begin{array}{l}\mathrm{N}=130 \\
\text { youngsters } \\
\text { with } 10-12 \\
\text { years old }\end{array}$ & $\begin{array}{l}\mathrm{N}=130 \\
\text { youngsters } \\
\text { with } 10-12 \\
\text { years old }\end{array}$ & No & $\begin{array}{l}\text { Quantitative } \\
\text { assessment }\end{array}$ & $\begin{array}{l}\text { Task and Ego } \\
\text { Orientation in } \\
\text { Sport } \\
\text { Questionnaire } \\
\text { (TEOSQ), } \\
\text { Perceived } \\
\text { Motivational } \\
\text { Climate in } \\
\text { Sport } \\
\text { questionnaire-2 } \\
\text { (PMCSQ-2), } \\
\text { Contextual } \\
\text { Self- } \\
\text { Responsibility } \\
\text { Questionnaire } \\
\text { (CSRQ) }\end{array}$ & No & $\begin{array}{l}\text { Descriptive } \\
\text { statistics of } \\
\text { observed } \\
\text { variables, } \\
\text { Simple } \\
\text { correlations } \\
\text { coefficients } \\
\text { among } \\
\text { observed } \\
\text { variables, } \\
\text { Path } \\
\text { analyses }\end{array}$ & $\begin{array}{l}\text { Task orientation and perceptions of a climate which } \\
\text { involved the task associated with the result, through } \\
\text { care for others and/ or goal setting, as well as self- } \\
\text { responsibility. The quality of participation was } \\
\text { influenced by the goals of the task. Guidance of ego } \\
\text { won't negatively impact contribute the model, maybe } \\
\text { due to the characteristics of the NYSP activities that } \\
\text { emphasized participation and physical activity. The } \\
\text { combination of nature involving the NYSP task and } \\
\text { the ages of the younger participants and limited } \\
\text { experience sport led to the partial attenuation of ego } \\
\text { orientation and your influence on motivation. } \\
\text { According to the analysis of the factor CSRQ } \\
\text { contained three factors: careful with the other goal } \\
\text { settings, self-responsibility and self-control/ respect. }\end{array}$ \\
\hline $\begin{array}{l}\text { Bean, } \\
\text { Kendelle } \\
\mathrm{n} \text {, and } \\
\text { Forneris } \\
(2016)\end{array}$ & $\begin{array}{l}\text { The purpose of this study } \\
\text { is to understand young } \\
\text { females perceptions of } \\
\text { life skills transference } \\
\text { and identify practical } \\
\text { strategies perceived by } \\
\text { young people effectively } \\
\text { facilitate the transfer. }\end{array}$ & $\begin{array}{l}\text { USA } \\
57 \\
\text { sessions }\end{array}$ & $\begin{array}{l}\text { After- } \\
\text { school } \\
\text { program }\end{array}$ & $\begin{array}{l}N=8 \\
12 \text { years old }\end{array}$ & $\begin{array}{l}N=8 \\
12 \text { years old }\end{array}$ & No & $\begin{array}{l}\text { Qualitative } \\
\text { assessment }\end{array}$ & Interview & No & $\begin{array}{l}\text { Inductive } \\
\text { analysis }\end{array}$ & $\begin{array}{l}\text { Female youngsters believed they were able to transfer } \\
\text { intrapersonal skills (emotional regulation, focus and } \\
\text { objectives), interpersonal (respect, responsibility and } \\
\text { social skills) and physical activity skills to contexts } \\
\text { beyond the program. One of the main strategies used } \\
\text { to intentionally teach life skills within the program } \\
\text { was used to present activities and provide } \\
\text { opportunities to practice the skills. }\end{array}$ \\
\hline
\end{tabular}

\section{Us Sociedad \\ Deporte}


Baptista, Corte-Real, Regueiras, Seo, Hemphill, Pereira, Dias, Martinek, Fonseca

\begin{tabular}{|c|c|c|c|c|c|c|c|c|c|c|c|}
\hline \multirow[b]{2}{*}{$\begin{array}{l}\text { Author(s)/ } \\
\text { year }\end{array}$} & \multirow[b]{2}{*}{ Focus } & \multirow[b]{2}{*}{$\begin{array}{l}\text { Local } \\
\text { /Duration } \\
\text { of the } \\
\text { program }\end{array}$} & \multirow[b]{2}{*}{$\begin{array}{l}\text { Context/ } \\
\text { Physical } \\
\text { activity }\end{array}$} & \multirow[b]{2}{*}{$\begin{array}{l}\text { Participants in } \\
\text { the program }\end{array}$} & \multicolumn{5}{|c|}{ Methodological design } & \multirow[b]{2}{*}{$\begin{array}{c}\text { Data } \\
\text { analysis }\end{array}$} & \multirow[b]{2}{*}{ Results } \\
\hline & & & & & $\begin{array}{l}\text { The study } \\
\text { sample }\end{array}$ & $\begin{array}{l}\text { Control } \\
\text { group }\end{array}$ & Study type & $\begin{array}{c}\text { Measurement } \\
\text { instrument }\end{array}$ & $\begin{array}{l}\text { Design } \\
\text { (pre } \\
\text { post } \\
\text { test) }\end{array}$ & & \\
\hline $\begin{array}{l}\text { Whitley, } \\
\text { and Gould } \\
\text { (2011) }\end{array}$ & $\begin{array}{l}\text { This article describes } \\
\text { a sport program for } \\
\text { refugee children and } \\
\text { youth in the United } \\
\text { States based on the } \\
\text { TPSR Model. }\end{array}$ & $\begin{array}{l}\text { USA } \\
8 \text { sessions }\end{array}$ & $\begin{array}{l}\begin{array}{l}\text { After-school } \\
\text { program }\end{array} \\
\text { Soccer, } \\
\text { Basketball, } \\
\text { Vollyball }\end{array}$ & Not reported & Not reported & No & $\begin{array}{l}\text { Not } \\
\text { reported }\end{array}$ & Not reported & No & Not reported & $\begin{array}{l}\text { Were described some lessons learned about how to } \\
\text { treat the young refugees seized as people and not as } \\
\text { athletes. The creation of rules in each group was } \\
\text { another lesson learned. } \\
\text { One of the major obstacles encountered was in the } \\
\text { communication the young refugees is not expressed } \\
\text { in English. Another obstacle was the wide disparity } \\
\text { of ages ( } 8-18 \text { years old). Issues have been developed } \\
\text { and directed to the different ages during the program. }\end{array}$ \\
\hline
\end{tabular}




\section{Personal and social responsibility model systematic review}

\section{Participants profiles (from sample)}

In this study, young people who teach physical activities to other young people and/ or children were called staff for being in the role of teachers but not being one, and to distinguish the name youngster, the young participants and children who received staff instruction.

Most youngsters (9/27) were considered vulnerable youngsters, due to drug abuse, use of violence, among other behaviors that may lead to social exclusion (Bean \& Forneris, 2015; Buckle \& Walsh, 2013; Hellison \& Wright, 2003; Martinek \& Schilling, 2003; Walsh et al., 2010; Whitley, 2011; Wright et al., 2012; Wright, 2012; Wright et al., 2012). The staff (10/27) were volunteers attending high school who willingly took part in intervention programs for the community (Hayden et al., 2012; Lee \& Martinek, 2009, 2012; Martinek \& Schilling, 2003; Martinek et al., 2006; Martinek et al., 2001; Schilling, 2001, 2007; Walsh, 2008; Hammond-Diedrich \& Walsh, 2006).

Regarding gender, there is a predominance (13/27) of interventions including both female and male (Beale, 2012; Forneris et al., 2013; Hammond-Diedrich \& Walsh, 2006; Hayden et al., 2012; Lee \& Martinek, 2009; Lee \& Martinek, 2012; Martinek et al., 2006; Newton et al., 2006; Schilling, 2001; Walsh, 2007; Walsh, 2008; Walsh et al., 2010; Whitley, 2011). However, there have been studies (3/27) targeted towards either one of genders, female (Bean \& Forneris, 2015; Schilling, 2007; Wright et al., 2012) or male 5/27 (Buckle \& Walsh, 2013; Coulson et al., 2012; Wright, 2012; Wright et al., 2012; Wright et al., 2004).

Some studies (5/27) did not report the genders of their participants (Buchanan, 2001; Forneris et al., 2013; Hellison \& Wright, 2003; Martinek \& Schilling, 2003; Martinek et al., 2001).

As for age, most interventions (11/27) focused on ages between 10 and 14 years (Bean \& Forneris, 2015; Forneris et al., 2013; Hammond-Diedrich \& Walsh, 2006; Hellison \& Wright, 2003; Lee \& Martinek, 2012; Newton et al., 2006; Schilling, 2001; Walsh, 2008; Walsh et al., 2010; Wright, 2012; Wright et al., 2012;). In some studies, the age of the participants ranged between 14 and 17 years (High school) (Beale,
2012; Martinek \& Schilling, 2003; Martinek et al., 2006), or younger ages, such as participants in elementary school (4/27) (Lee \& Martinek, 2009; Martinek et al., 2001; Walsh, 2007; Wright et al., 2012). Only two studies reported adult participants (Coulson et al., 2012; Schilling, 2007). Other studies reported several ages at the same time, from 12 to 17 years (Buckle \& Walsh, 2013); from 4 to 11 years (Wright et al., 2004), from 10 to 19 years (Forneris et al., 2013) or from 13 to 18 years (Forneris et al., 2013).

Some authors did not specify ages, referring to their participants as "Youngsters" in their studies (Buchanan, 2001; Hayden et al., 2012; Whitley, 2011).

When studies reported an intervention within the after school programs, the number of children was higher. However, it is important to note that the number of participants is directly linked to the number of staff who implemented the program. For example, in the study by Hayden et al. (2012), in which 110 youngsters were distributed into four groups (approximately 29 children in each group), with 12 trainers and 8 university students (staff) responsible for the implementation of the program, who, in turn, were distributed among the four groups, leading to five to eight children for each responsible technician, which literature considers a small group (a maximum of 15 to 20 participants per group) (Schilling, $2001 \mathrm{p}$. 356).

Other studies about after-school programs (8/27) maintained a small group of participants (HammondDiedrich \& Walsh, 2006; Lee \& Martinek, 2009; Lee \& Martinek, 2012; Martinek \& Schilling, 2003; Martinek et al., 2006; Martinek et al., 2001; Schilling, 2001; Walsh, 2008). This was also evident in interventions aimed towards vulnerable youngsters (7/27) (Bean \& Forneris, 2015; Walsh, 2007; Walsh et al., 2010; Whitley, 2011; Wright et al., 2012; Wright, 2012; Wright et al., 2012) or children with disabilities (Wright et al., 2004), with the exception of the studies (Beale, 2012; Buchanan, 2001; Hellison \& Wright, 2003), which reported larger sample sizes.

\section{Intervention background}

The fact that this research was directed towards after school interventions allowed us to observe a certain heterogeneity among the studies. The interventions as after school programs for community (leadership) 


\section{Baptista, Corte-Real, Regueiras, Seo, Hemphill, Pereira, Dias, Martinek, Fonseca}

where the students from high school prepared activities for children from disadvantaged communities that provided meaningful experiences in personal, social and sports settings for underserved children.

In most studies, the sample size agrees with Hellison (2003) and Schilling (2001); in other words, a small sample size (maximum of 20 participants). In case of an excessessive number of participants, they were divided into small groups during the intervention.

Often (9/27), the evaluation of the impact of the TPSR model was mainly focused on the staff (Hayden et al., 2012; Hammond-Diedrich \& Walsh, 2006; Lee \& Martinek, 2012; Martinek \& Schilling, 2003; Martinek, Schilling, \& Hellison, 2006; Martinek et al., 2001; Schilling, 2001; Schilling, 2007; Walsh, 2008) and seldom on the participants (Lee \& Martinek, 2009).

Club activities are another kind of intervention, these sports activities were normally aimed towards vulnerable youngsters (Buckle \& Walsh, 2013; Forneris et al., 2013; Hellison \& Wright, 2003; Whitley, 2011), and the activities consisted of clubs focusing on basketball (Coaching Club) (Walsh, 2007; Walsh et al., 2010) and martial arts (Kung Fu Club) (Wright, 2012; Wright et al., 2012), which took place over several months.

Summer Camps were types of programs characterized by lasting a short period of time, with a significant number of daily hours, and being aimed towards vulnerable youngsters. This type of intervention resorted to the use of fitness and team sports (Bean \& Forneris, 2015; Buchanan, 2001), sets of activities including golf, tennis, swimming, softball and soccer (Newton et al., 2006) or, simply, different types of physical, after-school activities (Wright et al., 2012).

In other contexts, the TPSR model was also found to be adapted to very particular backgrounds, such as a Life Guard course with a certificate at the end (Beale, 2012); associated to youngsters with special needs, such as in the context of recreational therapy (Coulson et al., 2012), and in the context of spastic diplegia cerebral palsy (Wright et al., 2004).

What research methodologies have been used to examine TPSR in after-school time settings?

\section{Type of methodology}

The use of a mixed methodology that combines qualitative and quantitative methods is the most recommended in literature for this type of studies and interventions (Gorard \& Makopoulou, 2012), as observed in 7/27 of the studies (Hayden et al., 2012; Hellison \& Wright, 2003; Martinek et al., 2006; Walsh, 2007; Walsh, 2008; Wright, 2012; Wright et al., 2012). Most of the studies analyzed were qualitative (12/27) (Bean et al., 2016; Beale, 2012; Buchanan, 2001; Hammond-Diedrich \& Walsh, 2006; Lee \& Martinek, 2009; Lee \& Martinek, 2012; Martinek \& Schilling, 2003; Martinek et al., 2001; Schilling, 2001; Schilling, 2007; Walsh et al., 2010; Wright et al., 2004) and only two studies used a quantitative methodology (Bean \& Forneris, 2015; Newton et al., 2006).

In some studies, there was no mention to the methodology used (6/27) (Buckle \& Walsh, 2013; Coulson et al., 2012; Forneris et al., 2013; Whitley, 2011; Whitley \& Gould, 2011; Wright et al., 2012).

The lack of scientific rigor in study methodology can be verified (Q. 3, Table 1) by the large number of studies that failed to report the validity and reliability $(15 / 27)$ or just mentioned without great detail (6/27), as well as by the absence or lack of methods (6/27) and a detailed description of the methodology used in the studies $(9 / 27)$.

What TPSR-related outcomes were experienced by students in after school time settings?

\section{Outcomes}

Several studies (10/27) mentioned improvements of personal and social skills, self-control, leadership. However, they didn't report how was data operationalized (Beale, 2012; Buckle \& Walsh, 2013; Coulson et al., 2012; Martinek et al., 2006; Walsh, 2007; Wright et al., 2012; Wright et al., 2012; Whitley, 2011; Forneris et al., 2013; Whitley \& Gould, 2011).

The results were grouped according to the characteristics of the analyzed studies, based on concepts intrinsic to the TPSR model, such as personal outcomes, social outcomes, leadership, relationship between youngsters and staff, values, transference and impact on the young people's lives. 


\section{Personal and social responsibility model systematic review}

Personal outcomes - An improvement in young people's behavior, such as paying more attention in class and in their lives, was reported by Walsh (2007). In other studies, the personal outcomes were evidenced when ten of the thirteen participants created their own goals within and outside the program (Walsh et al., 2010), as well as, when the young people complied with the pre-established goals (Martinek et al., 2001; Schilling, 2007), thus helping them to cope with dysfunctional problems at home (Martinek \& Schilling, 2003), which led to academic improvement for $75 \%$ of the participants (Wright et al., 2012).

In the particular case of Tasha (Schilling, 2007), over time, she became more independent and improved her parental functions (teenage pregnancy). Although she may have not fully achieved the personal and social skills, there was an improvement described in her own self-reporting and in the view of the researchers, in terms of her behavior and attitudes towards herself and others.

On the other hand, there was also a decrease of personal responsibility, although it was not statistically significant, at the end of the intervention (Bean \& Forneris, 2015). Another study (Walsh, 2007), which compared youngsters development outcomes between young people who participated in the TPSR-based program and students from the same school who had not participated in the program, showed that students who had not participated in the program reported (statistically significant, $\mathrm{p}<0.05$ ) school being an even greater challenge, when they were approached regarding high expectations.

Social outcomes - There were studies that revealed an increase in the perception of social responsibility at the end of the intervention (Bean \& Forneris, 2015), which was also proven in young people with disabilities (Wright et al., 2004). The participants exhibited self-control (Buchanan, 2001; HammondDiedrich \& Walsh, 2006; Martinek et al., 2001) by improving their behavior at school $(62 \%$ of participants) and by becoming more mature (Hammond-Diedrich \& Walsh, 2006).

Another notable example of the acquisition of social skills was reported in the study by Whitley (2011), in which the young people at the end of the intervention gave more hi-fives and less negative feedback to their teammates. Care for others and willingness towards team work was also well evidenced in the studies by Martinek et al. (2001) and Walsh et al. (2010).

On the other hand, in interventions with a shorter duration, it became apparent that, although young people exhibited some changes in their behavior, they still manifested behaviors such as not respecting their teammates by laughing at others (Buchanan, 2001).

Leadership - Leadership ability is a very present concept in the TPSR. Some studies reported a development of this ability (Hayden et al., 2012) as a great opportunity for young people to outperform (Martinek \& Schilling, 2003). Often, the concept of leadership was intrinsic to the nature of the intervention program, such as the case of the staff who had to autonomously prepare and teach the sports activities to the children (8/27) (Hammond-Diedrich \& Walsh, 2006; Hayden et al., 2012; Lee \& Martinek, 2009; Lee \& Martinek, 2012; Martinek \& Schilling, 2003; Martinek et al., 2006; Schilling, 2001; Walsh, 2008).

Relationship between staff and youngsters - The relationships established between the staff from university and the staff from high school (4/27) (Buckle \& Walsh, 2013; Hellison \& Wright, 2003; Schilling, 2001; Walsh et al., 2010), between peers (Wright, 2012), or between staff and youngsters (Schilling, 2001) were highlighted as a very positive aspect of the program, with a mutual benefit (Hammond-Diedrich \& Walsh, 2006; Martinek et al., 2006), regardless of age (Hammond-Diedrich \& Walsh, 2006; Wright et al., 2004). This relationship was strengthened by the care and concern towards youngters' lives, which motivated them not to give up (Buckle \& Walsh, 2013) on their goals and made them aware of the importance of taking care of their wellbeing and the well-being of others (Hellison \& Wright, 2003; Martinek \& Schilling, 2003). The strong relationships made a difference in the most underserved children in the community (Martinek \& Schilling, 2003).

Values - In the interventions, youngsters stated that the TPSR model was a facilitator of role-modeling behavior, as it was based on values such as respect for the rights and feelings of others (Schilling, 2001). Participants also showed to be more aware of the use of these skills (values) outside the program (Wright et al., 2012). Paul Wright, in his study (Wright, 2012), 


\section{Baptista, Corte-Real, Regueiras, Seo, Hemphill, Pereira, Dias, Martinek, Fonseca}

reported that $70 \%$ of participants evidenced improvement regarding certain values such as honesty, care, respect and responsibility.

The participation of youngsters in value-based and task-based programs rather than ego-based programs has generally led to the development of responsibility in staff (Hammond-Diedrich \& Walsh, 2006; Newton et al., 2006).

Transference - It is relevant to point out that the transfer of the values learned in the sessions is grasped and applied outside the context of the session (Hellison, 2011).

Some studies (8/27) focused on the theme download, identifying benefits and results as the transference of the values learned in the sessions to after schoolsettings. It was found that $62 \%$ of participants demonstrated a medium and high level of transference (Martinek et al., 2001). In other analyzed studies, participants reported that the program would help them both within and outside the intervention (Beale, 2012; Forneris et al., 2013; Hammond-Diedrich \& Walsh, 2006; Lee \& Martinek, 2012; Walsh et al., 2010), as these youngsters became better individuals, and not only better basketball players (Hellison \& Wright, 2003), since that transference would aid them in decision making, creating aspirations and making choices for the future (Walsh, 2008).

On the other hand, two studies reported that the transference was the least visible level (subject) during the interventions (Hayden et al., 2012), and that culture, for example, due to its characteristics, also functioned as a barrier to the transference of the program values to the school setting, as is evidenced in the study by Lee and Martinek (2009), where the empowerment values in Project Effort (e.g., respect, responsibility) were perceived as a discipline approach in the school setting.

Although, there is no knowledge in the literature of instruments that measure the perception of transfer, in some cases (6/27) this was considered for interviews (Hammond-Diedrich \& Walsh, 2006; Hayden et al., 2012; Lee \& Martinek, 2009, 2012; Walsh, 2008; Walsh et al., 2010).

Impact on staff - A positive impact of the program on the lives of the participants, as well as their respective communities, was reported by Forneris et al. (2013). The staff described the program as safe (Whitley, 2011), fun (Hammond-Diedrich \& Walsh, 2006; Forneris et al., 2013; Schilling, 2001; Wright et al., 2012) - even more than traditional therapy (Wright et al., 2004) -, and that the content (martial arts) was in line with TPSR-based values TPSR (Wright et al., 2012; Wright et al., 2004).

Through the TPSR program, staff improved their teaching skills with children and became more familiar with college (intervention location) (Hammond-Diedrich \& Walsh, 2006). Individual experiences during the interventions were reported by staff as very significant when they led a group of children, and when they worked in peer-coaching (Hammond-Diedrich \& Walsh, 2006; Wright et al., 2012), because it allowed them to learn with their peers (Forneris et al., 2013).

Other evidence was reported by staff when they conveyed the desire to spend more time in the program (Hammond-Diedrich \& Walsh, 2006; Walsh, 2007). With assiduous participation in the program, staff reported that it provided them with opportunities to follow through with their commitments (Schilling, 2001; Whitley, 2011).

In a more longitudinal view, the instructor with experience in leadership programs continued with TPSR-based teaching in her professional life, she was guided by values and was enthusiastic about passing them on to the children after observing positive changes in their behavior (Coulson et al., 2012).

\section{DISCUSSION}

The purpose of this study was to conduct a systematic literature review about programs based on the Teaching Personal and Social Responsibility (TPSR) model in after-school settings. This study aimed to fulfill the absence of a systematic review based on the TPSR model in after-school settings.

Similar to other more recent review studies about the TPSR model, such as Casey (2014); Caballero-Blanco et al. (2013) and Pozo et al. (2016), this study followed a protocol for systematic reviews (Coharance), obeying a specific method and selection criteria. 


\section{Personal and social responsibility model systematic review}

Most implementations were based on after-school programs for community and continued being conducted in the United States of America, as reported by Caballero-Blanco et al. (2013). As mentioned in the introduction, TPSR model has been applied in different parts of the world. It would be interesting to apply the TPSR model also in after-school context as in intervention programs for the community, in clubs, or at summer camps involving both genders in different countries.

Regarding the design of the interventions, there was a superiority of qualitative methods, as evidenced by Caballero-Blanco et al. (2013); Hellison and Walsh (2002), and Pozo et al. (2016).

The lack of systematization in the studies, specifically in methodologic design, was evident by the lack of validity and reliability the absence of a detailed description of the methods used not to mention, in some studies as the analysis of the data. No study of qualitative nature mentioned what software is used for the treatment of the data.

It would be interesting to fill this gap in future studies. More robust and sophisticated methodologies and instruments must be developed to assess the real impact on the development of intervention programs, as in the case of the TPSR model. Scientific rigor in methodology is a critical step for the advancement of literature in TPSR model-based interventions in afterschool contexts.

Regarding the outcomes, it was found that after-school programs based on the Hellison model, as CaballeroBlanco et al. (2013) reported in his review study, also provided a significant increase of personal and social responsibility. This finding was statistically demonstrated through interviews and direct behavior observation. The development of personal and social responsibility, as well as other values is, naturally, less effective in short-term implementations.

The transference of values, learned and developed during the program, despite difficult visibility, was found in some studies (Hellison \& Wright, 2003; Walsh, 2008). It would be interesting to know what aspects of the intervention contributed to these changes.
The programs based on the TPSR model, due to its particular characteristics, provided opportunities for staff and youngsters to disclose and share their fears, concerns, and goals (Walsh, 2008). This is facilitated by a strong relationship between members of the program and participants, which was often referred to as an important and determinant aspect in the lives of staff from high school. This relationship was also characterized by a psychologically and physically secure environment, as reported in another review study by Pozo et al. (2016).

FInally, the after-school interventions based on the TPSR model provided significant experiences (especially in leadership) and had a positive impact on staff and youngsters who engaged in sports activities (5/27) (Bucke \& Walsh, 2013; Martinek et al., 2006; Walsh, 2007; Wright et al., 2012; Hammond-Diedrich \& Walsh, 2006).

\section{Future directions}

As suggested in previous review studies, (CaballeroBlanco et al., 2013; Hellison and Walsh, 2002, and Pozo et al., 2016), more studies with mixed methodologies (qualitative and quantitative), and longitudinal studies are required that can intersect several implementations of the same project, such as Project Effort (Martinek \& Schilling, 2003), in order to further understand the impact of after-school programs on the lives of young people and on society in general.

Although the examined studies refer in detail to the description of the intervention program (Q1, Table 1), further studies that apply scientifically rigorous methodological design, with a more detailed description of the methods, using the validity and reliability of the study, with pre- and post-design tests are needed.

\section{REFERENCES}

1. Baptista, C., Bessa, C., Pereira, S., Corte-Real, N., Regueiras, L., \& Maia, J. (2016). Adaptação de um programa da responsabilidade pessoal e social na formação de professores: Um estudo piloto em Timor-Leste. VERITAS, 4(2), 5-16.

2. Beale, A. (2012). Fulfilling the promise of making a difference: Creating guards of life with TPSR. 


\section{Baptista, Corte-Real, Regueiras, Seo, Hemphill, Pereira, Dias, Martinek, Fonseca}

International Journal of Aquatic Research and Education(6), 249-266.

3. Bean, C., \& Forneris, T. (2015). Using a timeseries analysis to evaluate a female youth-driven physical activity-based life skills program based on the teaching personal and social responsibility model. Ágora para la EF y el Deporte, 12(2), 94114.

4. Bean, C., Kendellen, K., \& Forneris, T. (2016). Moving beyond the gym exploring life skill transfer within a female physical activity based life skills program. Journal of Applied Sport Psychology, 1-17. doi:10.1080/10413200.2015.1124155

5. Belando, N., Ferriz-Morell, R., \& MorenoMurcia, J. (2012). Propuesta de un modelo para la mejora personal y social a través de la promoción de la responsabilidad en la actividad físicodeportiva. Revista Internacional de Ciencias del Deporte, 8(29), 202-222. doi:10.5232/ricyde2012.02902

6. Blanco, P. (2015). Percepción del alumnado de formación profesional sobre los efectos de un programa de desarrollo positivo (modelo de responsabilidad de Hellison). Journal of Sport and Health Research, 7(2), 113-126. doi: $10.4321 / \mathrm{s} 1578-84232015000200020$

7. Buchanan, A. (2001). Contextual challenges to teaching responsibility in a sports camp. Journal of Teaching in Physical Education (20), 155-171.

8. Buckle, M., \& Walsh, D. (2013). Teaching responsibility to gang-affiliated youths. Journal of Physical Education, Recreation \& Dance, 84(2), 53-58. doi:10.1080/07303084.2013.757193

9. Caballero-Blanco, P., Delgado-Noguera, M., \& Escartí, A. (2013). Analysis of teaching personal and social responsibility model-based programmes applied in USA and Spain. Journal of Human Sport \& Exercise, 8(2), 427-441. doi:10.4100/jhse.2012.82.10

10. Camerino, O., Valero-Valenzuela, A., Prat, Q., Manzano Sanchez, D., \& Castaner, M. (2019). Optimizing Education: A Mixed Methods Approach Oriented to Teaching Personal and Social Responsibility (TPSR). Frontiers in Psychology, 10, 1439.
11. Casey, A. (2014). Models-based practice: great white hope or white elephant? Physical Education and Sport Pedagogy, 19(1), 18-34. doi:10.1080/17408989.2012.726977

12. Catalano, R., Berglund, M., Ryan, J., Lonczak, H., \& Hawkins, J. (2004). Positive youth development in the United States: Research findings on evaluations of positive youth development programs. The Annals of the American Academy of Political and Social Science, 591, 98-124. doi:10.1177/0002716203260102

13. Coulson, C., Irwin, C., \& Wright, P. (2012). Applting Hellison's responsibility model in a youth residential treatment facility: a practial inquiry project. Ágora para la EF y el Deporte, l(14), 38-54.

14. Díaz, J., Manzano, J., Martín, M., Catalán, J., \& Palacios, A. (2018). Formación en valores desde el Área de Psicología del Deporte del Sevilla F.C. S.A.D. Revista de Psicología Aplicada al Deporte y el Ejercicio Físico, 3(1), 1-8. doi:10.5093/rpadef2018a5

15. Dunn, R., Hemphill, M., \& Beaudoin, S. (2006). A community of practice that supported the transition from doctoral student to faculty member. Journal of Physical Education, Recreation \& Dance, 85(5), 27-30. doi:10.1080/07303084.2016.1157391

16. Escartí, A., Gutiérrez, M., Pascual, C., \& Wright, P. (2013). Observación de las estrategias que emplean los profesores de educación física para enseñar responsabilidad personal y social. Revista de Psicología del Deporte, 22(1), 159-166. doi:10.5232/ricyde2011.02203

17. Escartí, A., Wright, P., Pascual, C., \& Gutiérrez, M. (2015). Tool for assessing responsibility-based education (TARE) 2.0: instrument revisions, interrater reliability, and correlations between observed teaching strategies and student behaviors. Universal Journal of Psychology, 3(2), 55-63. doi:10.13189/ujp.2015.030205

18. EndNote X7, 2014. Thompson Reuters.

19. Fernández-Ríos, L., \& Buela-Casal, G. (2009). Standards for the preparation and writing of psychology review article. International Journal of Clinical and Health Psychology, 9(2), 329-344. 


\section{Personal and social responsibility model systematic review}

20. Forneris, T., Whitley, M., \& Barker, B. (2013). The Reality of Implementing community based sport and physical activity programs to enhance the development of underserved youth: Challenges and potential strategies. Quest, 65(3), 313-331. doi:10.1080/00336297.2013.773527

21. Gorard, S., \& Makopoulou, K. (2012). Is mixed methods the natural approach to research? In K. Armour \& D. Macdonald (Eds.), Research methods in physical education and youth sport (pp. 106-119). London and New York: Routledge.

22. Gordon, B., \& Doyle, S. (2015). Teaching personal and social responsibility and transfer of learning: opportunities and challenges for instructors and coaches. Journal of Teaching in Physical Education, 34(1), 152-161. doi:10.1123/jtpe.2013-0184

23. Hammond-Diedrich, K., \& Walsh, D. (2006). Empowering youth through a responsibility-based cross-age instructor program: An investigation into impact and possibilities. The Physical Educator, 63(3).

24. Hayden, L., Baltzell, A., Kilty, K., \& McCarthy, J. (2012). Developing responsibility using physical activity: A case study of team support. Ágora para la EF y el Deporte, 14(2264-281).

25. Hellison, D. (1985). Goals and strategies for physical education. Champaign, IL: Human Kinetics.

26. Hellison, D. (1995). Teaching responsibility through physical activity ( 1 ed.). Champaign, IL: Human Kinetics.

27. Hellison, D. (2003). Teaching responsibility through physical activity (2 ed.). University of Illinois at Chicago: Human Kinetics.

28. Hellison, D. (2011). Teaching Personal and Social Responsibility Trough Physical Activity (3 ed.). USA: Human Kinetics.

29. Hellison, D., \& Martinek, T. (2006). Social and Individual responsibility programs. In D. Kirk, M. O'Sullivan, \& D. Macdonald (Eds.), Handbook of research in physical education (pp. 610-626). London: Sage.

30. Hellison, D., \& Walsh, D. (2002). Responsibilitybased youth programs evaluation: Investigating the investigations. Quest (54), 292-307.
31. Hellison, D., \& Wright, P. (2003). Retention in an Urban Extended Day Program: A Process-Based Assessment. Journal of Teaching in Physical Education, 22(4), 369-381. doi:10.1123/jtpe.22.4.369

32. Higgins, J. P., \& Green, S. (Eds.). (2011). Cochrane Handbook for Systematic Reviews of Interventions (Version 5.1.0 ed.): www.handbook.cochrane.org.

33. Landis J., \& Koch G. (1977). The measurement of observer agreement for categorical data. Biometrics, 33, 159-174.

34. Lee, O., \& Choi, E. (2015). The influence of professional development on instructors' implementation of the teaching personal and social responsibility model. Journal of Teaching in Physical Education, 34(4), 603-625. doi:10.1123/jtpe.2013-0223

35. Lee, O., \& Martinek, T. (2009). Navigating two cultures: An investigation of cultures of a responsibility-based physical activity program and school. Research Quarterly for Exercise \& Sport, $80, \quad 230-240$. doi:10.1080/02701367.2009.10599557

36. Lee, O., \& Martinek, T. (2012). Factors influencing transfer of responsibility-based physical activity program goals into classrooms. The Physical Educator(69), 188-207.

37. Martinek, T. (2009). Project effort: Serving the underserved populations through sport. Kinesiology Today, 2(2), 4-8.

38. Martinek, T. (2016). Project effort: Creating responsible leaders through values-based learning. Active, Helthy Magazine.

39. Martinek, T., \& Schilling, T. (2003). Developing Compassionate Leadership in Underserved Youths. Journal of Physical Education, Recreation \& Dance, 74(5), 33-39. doi:10.1080/07303084.2003.10608483

40. Martinek, T., Schilling, T., \& Hellison, D. (2006). The development of compassionate and caring leadership among adolescents. Physical Education and Sport Pedagogy, 11(2), 141-157.

41. Martinek, T., Schilling, T., \& Johnson, D. (2001). Transferring personal and social responsibility of 


\section{Baptista, Corte-Real, Regueiras, Seo, Hemphill, Pereira, Dias, Martinek, Fonseca}

undersevered youth to the classroom. The Urban Review, 33(1).

42. Metzler, M. (2005). Instructional models for physical education. Boston: MA: Allyn \&Bacon.

43. Moher, D., Schulz, K., and Altman, D. (2001). The CONSORT statement: Revised recommendations for improving the quality of reports of parallel-group randomised trials. Lancet 357(9263): 1191-1194

44. Newton, M., Watson, D., Kim, M.-S., \& Beacham, A. (2006). Understanding motivation of underserved youth in physical activity settings. Youth \& Society, 37(3), 348-371. doi:10.1177/0044118X05278964

45. Pan, Y.-H., \& Keh, N.-C. (2014). Teaching responsibility through physical education: Research and applications in Taiwan. Japanese Journal of Sport Education Studies, 34(1), 63-69.

46. Petitpas, A., Cornelius, A., Raalte, J. V., \& Jones, T. (2005). A Framework for planning youth sport programs that foster psychosocial development. Human Kinetics Publishers, (19), 63-80.

47. Pozo, P., Grao-Cruces, A., \& Pérez-Ordás, R. (2016). Teaching personal and social responsibility model-based programmes in physical education. European Physical Education Review, 1-20. doi:10.1177/1356336x16664749

48. Prieto, J., Delgado, F., Caro, L. \& Preciado, A. (2015). Desarrollo de los componentes sociales de la deportividad en futbolistas alevines. Cuadernos de Psicología del Deporte, 15(2), 113-124. doi: $10.4321 / \mathrm{S} 1578-84232015000200013$

49. Schilling, T. (2001). An investigation of commitment among participants in an extended day physical activity program. Research Quarterly for Exercise and Sport, 72(4), 355-365. doi:10.1080/02701367.2001.10608972

50. Schilling, T. (2007). An Examination of Resilience Processes in Context: The Case of Tasha. The Urban Review, 40(3), 296-316. doi:10.1007/s11256-007-0080-8

51. Shea, B., Grimshaw, J., Wells, G., Boers, M., Andersson, N., Hamel, C., . . Bouter, L. (2007). Development of AMSTAR: a measurement tool to assess the methodological quality of systematic reviews. BMC Med Res Methodol, 7, 10. doi:10.1186/1471-2288-7-10

52. Siegel S, \& Castellan N. (1988). Nonparametric statistic for the behavioral sciences (2 ed.). New York: McGraw-Hill.

53. Vandenbroucke, J., Elm, E., Altman, D., Gøtzsche, P., Mulrow, C., Pocock, S., . . Egger, M. (2007). Strengthening the reporting of observational studies in epidemiology (STROBE): Explanation and elaboration. Annals of Internal Medicine, 147(6), 163-194.

54. Walsh, D. (2007). Supporting youth development outcomes: An evaluation of a responsibility model-based program Physical Educator, 64(1), 48-56.

55. Walsh, D. (2008). Helping youth in underserved communities envision possible futures: An extension of the teaching personal and social responsibility model. Research Quarterly for Exercise and Sport, 79(2), 209-221. doi:10.1080/02701367.2008.10599484

56. Walsh, D., Veri, M., \& Willard, J. (2015). Kinesiology career club: Undergraduate student mentors' perspectives on a physical activitybased teaching personal and social responsibility program. The Physical Educator, 72, 317-339.

57. Walsh, D., Ozaeta, J., \& Wright, P. (2010). Transference of responsibility model goals to the school environment: exploring the impact of a coaching club program. Physical Education \& Sport Pedagogy, 15(1), 15-28. doi:10.1080/17408980802401252

58. Walsh, D., \& Wright, P. (2016). The TPSR Alliance a community of practice for teaching research and service. Journal of Physical Education, Recreation \& Dance, 87(5), 35-38. doi:10.1080/07303084.2016.1157394

59. Whitley, M. (2011). The Ikhaya sport programs in the Kayamandi township. Ágora, 14(1), 115-136.

60. Whitley, M., \& Gould, D. (2011). Psychosocial Development in Refugee Children and Youth through the Personal-Social Responsibility Model. Journal of Sport Psychology in Action, 1(3), 118-138. doi:10.1080/21520704.2010.534546 
61. Wright, E., Whitley, M., \& Sabolboro, G. (2012). Conducting a TPSR program for an underserved girl's summer camp. Ágora para la EF y el Deporte, 14(1), 5-24.

62. Wright, P. (2012). Offering a TPSR physical activity club to adolescent boys labeled "at risk" in partnership with a community-based youth serving program. Ágora para la EF y el Deporte, 14(2), 94-114.

63. Wright, P., Dyson, B., \& Moten, T. (2012). Exploring the individualized experiences of participants in a responsibility-based youth development program. Ágora para la EF y el Deporte, 2(14), 248-263.

64. Wright, P., Jacobs, J., Ressler, J., \& Jung, J. (2016). Teaching for transformative educational experience in a sport for development program. Sport, Education and Society, 21(4), 531-548. doi:10.1080/13573322.2016.1142433

65. Wright, P., White, K., \& Gaebier-Spira, D. (2004). Exploring the relevance of the personal and social responsibility model in adapted physical activity: A collective case study. Journal of Teaching in Physical Education, 23(1), 71-87. doi:10.1123/jtpe.23.1.71 